\title{
Medicinal Utility of Pomegranate Fruit in Regular Human Diet: A Brief Review
}

\author{
Subha Ganguly \\ Associate Professor, Department of Veterinary Microbiology, ARAWALI VETERINARY \\ COLLEGE (Affiliated with Rajasthan University of Veterinary and Animal Sciences, Bikaner), \\ Rajasthan, India \\ ganguly38@gmail.com
}

\begin{abstract}
Pomegranate in Hindi is called Anar and Beejpur in Sanskrit. It is being in wide application for thousands of years as a curative against many diseases across different cultures and civilizations. The fruit possess tremendous nutritional values and medicinal health benefits. In India the root, bark, flowers, fruit and leaves of the Pomegranate are used in traditional ayurvedic therapy and medicinal applications. The plant bears the fruits from September to February in the the Northern Hemisphere and during March to May in Northern hemisphere. The fruit has multivarious applications in food processing industries in meal garnishes, smoothies, baking, cooking, juice blends and in alcoholic beverages.
\end{abstract}

Keywords: Ayurveda, Pomegranate, Traditional medicine.

\section{INTRODUCTION}

The pomegranate fruit is botanically known as Punica granatum, The small plant is a deciduous shrub bearing fruits and is categorized under the family Lythraceae. It attains a height between 5 and $8 \mathrm{~m}$.

\section{Nutritional Importance}

Nutritionally pomegranate seeds are rich source of Vitamin C, Vitamin K and folate. It acts as an rich source of dietary fibre and are edible in nature. It also supplies micronutrients to the feeders.

The seed oil contains punicic acid (65.3\%), palmitic acid (4.8\%), stearic acid (2.3\%), oleic acid $(6.3 \%)$, and linoleic acid $(6.6 \%) .{ }^{[1-3]}$

\section{Juice}

The pomegranate juice contains abundant phytochemicals like polyphenols, including the hydrolyzable tannins called ellagitannins. The fruit juice is red in color for the presence of anthocyanins, such as delphinidin, cyanidin, and pelargonidin glycosides. As the fruit ripens there is an increase in the redness in its juice. It is always recommended to consume the whole fruit by chewing rather than having the processed and packaged juice sterilized by pasteurization, as it leads $t$ the decline in food value.

Peel

The peel of the pomegranate contains as much as three times the total amount of polyphenols, which includes catechins, gallocatechins condensed tannins and prodelphinidins as compared to the fruit pulp.

The higher phenolic content of the peel yields extracts for use in dietary supplements and food preservatives.

\section{Medicinal Properties ${ }^{[4-6]}$}

Cancer: Pomegranates consist of advanced level of antioxidants called flavenoids. These flavenoids are thought to be effective in counteracting various cancer radials. Regular intake of the fruit juice lessens the high risk of prostate and breast cancer and in combating the existing cancer cells in the body.

Heart Problems: Regular intake if the fruit helps in maintaining good blood flow in the body. It also decreases the risks of cardiac arrests and heart strokes. 
Stomach Disorder: Pomegranates peel, bark and leaves are effectively employed for the curing of digestive disorders leading to diarrhea. Pomegranate juice is also used for treatment of dysentery and cholera.

Osteoarthritis: Pomegranate acts as a remedy in the cure of illness triggered in various forms, like atherosclerosis and osteoarthritis caused by the thickening and solidifying of the arterial walls and in cartilage and joints.

Diabetes: The juice of pomegranate helps in the prevention of coronary illnesses in diabetic patients.

Dental Care: Pomegranate juice possesses antibacterial and antiviral properties which are used effectively in the reduction of incidences of dental plaque.

Anemia: Pomegranate seed extract supplies iron to blood and thus, helps in decreasing the occurrence of anemic symptoms including weakness, fatigue, and hear loss.

Other Proven Uses ${ }^{[7-9]}$

The other advantages of pomegranate fruit include reduction in the occurrence of premature infants and it is also beneficial for the expected mothers to avoid having low weight infants during birth. Among the elder people, regular intake of pomegranate reduces the likelihood of creating Alzheimer's disease. It helps in controlling aging issues thereby sustaining youthful and glowing skin. During menopause period, consumption of pomegranate allows a woman to overcome from her depression interval. It is a good natural aphrodisiac and improves sperm count and semen quality.

The pomegranate flower, juice, rind and tree bark also possess astringent properties and are valuable for a wide range of purposes, such as stopping nose bleeds and gum bleeds and in treating hemorrhoid.

\section{CONCLUSION}

Pomegranate seed (of specific fruit strains) is also used as eye drops as it is believed to slow the development of cataracts. Pomegranate is used as a gargle for a sore throat, and it is applied to the epidermis to cure hemorrhoid flare-ups. It cleanses and clarifies oral cavity, throat, esophagus stomach and chest.

\section{REFERENCES}

[1] LaRue, James, H. (1980). Growing Pomegranates in California. California Agriculture and Natural Resources. Retrieved 2007-10-25.

[2] Morton JF (1987). Pomegranate, Punica granatum L. Fruits of Warm Climates. Purdue New Crops Profile, pp. 352-5

[3] Chidambara Murthy, K.N., Jayaprakasha, G.K. and Singh, R.P. (2002). Studies on Antioxidant Activity of Pomegranate (Punica granatum) Peel Extract Using in Vivo Models. Journal of Agricultural and Food Chemistry, 50(17): 4791. doi:10.1021/jf0255735

[4] Li, Y., Guo, C., Yang, J., Wei, J., Xu, J. and Cheng, S. (2006). Evaluation of antioxidant properties of pomegranate peel extract in comparison with pomegranate pulp extract. Food Chemistry, 96 (2): 254.doi:10.1016/j.foodchem.2005.02.033

[5] Negi, P.S., Jayaprakasha, G.K. and Jena, B.S. (2003). Antioxidant and antimutagenic activities of pomegranate peel extracts. Food Chemistry, 80 (3): 393.doi:10.1016/S0308-8146(02)00279-0

[6] Plumb, G.W., De Pascual-Teresa, S., Santos-Buelga, C., Rivas-Gonzalo, J.C. and Williamson, G. (2002). Antioxidant properties of gallocatechin and prodelphinidins from pomegranate peel. Redox Rep., 7(41): 41-6.doi:10.1179/135100002125000172

[7] Hernández, F., Melgarejo, P., Tomás-Barberán, F.A. and Artés, F. (1999). Evolution of juice anthocyanins during ripening of new selected pomegranate (Punica granatum) clones. European Food Research and Technology, 210 (1): 39-42.doi:10.1007/s002170050529

[8] Jayaprakasha, G.K., Negi, P.S. and Jena, B.S. (2006). Antimicrobial activities of pomegranate. In: Seeram, Navindra P., Schulman, Risa N. and Heber, David. Pomegranates: ancient roots to modern medicine. CRC Press. pp. 168. ISBN 978-0-8493-9812-4.

[9] Stover, E. and Mercure, E.W. (2007). The pomegranate: a new look at the fruit of paradise. Hort. Science, 42 (5): 1088-92. 